\title{
OPEN ACCESS UND DAS HYBRIDE ZEITSCHRIFTENMODELL - EINE ANALYSE ZUR TRANSPARENZ BEI AUSGEWÄHLTEN VERLAGEN
}

\author{
von Sabine Stigler
}

\section{Inhalt}

1. Hybride Journale und die Problematik des Double Dippings

2. Ergebnisse der webseitenbasierten Analyse zur Preisanpassung bei hybriden Journalen

3. Empfehlungen zu mehr Transparenz

4. Befragung der Verlage und Ergebnisse

5. Zusammenfassung

Zusammenfassung: Das hybride Publikationsmodell bietet die Möglichkeit, Artikel in Subskriptionszeitschriften gegen Gebühr freizukaufen und damit für alle zugänglich zu machen. Gegenstand des Beitrages (basierend auf der Masterthesis mit gleichnamigem Titel) sind die hybriden Journale sieben ausgewählter Verlage. Die Aussagen der Interviews, die im Zuge der Masterthesis durchgeführt wurden, sowie die Webseitenanalyse stellen den Kern dieses Beitrages dar. Ziel ist es, Intransparenz aufzudecken und Empfehlungen zu geben, wie mehr Transparenz ermöglicht werden könnte. Hierbei sollen Aussagen zu den dringlichsten Fragen, u.a. ob und wie das hybride Modell fortbestehen wird, und wie die Anpassung der Subskriptionspreise am besten gehandhabt werden kann, geklärt werden. Darüber hinaus werden Vorschläge präsentiert, welche Parameter die Listen zur Preisanpassung beinhalten sollten, damit mehr Transparenz gewährleistet werden kann.

Schlüsselwörter: Open Access; hybride Journale; Double Dipping; Verlage; Anpassung der Subskriptionspreise

\section{OPEN ACCESS AND THE HYBRID JOURNAL PUBLISHING MO- DEL - AN ANALYSIS REGARDING TRANSPARENCY OF SELECTED PUBLISHERS}

Abstract: The hybrid model gives authors the opportunity to purchase their articles in a subscription journal in order to make it freely available for others. The hybrid journals of seven publishers are the main focus of this work. Interviews with the publishers and the adjustment lists of subscription journals on their websites form the basis of the analysis presented here. The aim of this contribution is to uncover opacity and to give recommendations as to how a higher degree of transparency could be achieved. 
This study evaluates the publishers' websites. In addition, the interviews conducted with the publishers provide answers to the most urgent questions, e.g. will they continue the hybrid model. In conclusion, this article offers some suggestions as to which parameters would be needed in order to achieve greater transparency for the lists of price adjustments published.

Keywords: Open Access; hybrid journals; Double Dipping; publishers; adjustment of subscription prices

\section{Hybride Journale und die Problematik des Double Dippings}

Hybride Journale geben Autorlnnen die Möglichkeit, ihre wissenschaftlich generierten Inhalte gegen die Zahlung einer Gebühr (APCs) freizukaufen und somit Endnutzerlnnen, die keine Subskription des Journals haben, kostenfrei zur Verfügung zu stellen. Die Anzahl von hybriden Journalen hat sich in den letzten Jahren verdoppelt und lag 2012 bei ungefähr 4.300 (vgl. Björk, 2012), während die Anzahl der Artikel, die auf diesem Weg frei zugänglich gemacht wurden, im Jahr 2011 ungefähr 12.000 betrug (vgl. Björk, 2012). Laut Björk hat sich die Akzeptanz dieser Option inzwischen eingependelt und liegt bei 1-2\% der Gesamtanzahl an erschienenen Artikeln. Davon abweichende Zahlen ergeben sich dadurch, dass sich nicht die Anzahl an Autorlnnen, die die hybride Option in Anspruch genommen haben, sondern vielmehr das Portfolio an Zeitschriften, die diese Option anbieten, in den letzten Jahren vergrößert hat (vgl. ebd.).

Die gestiegene Anzahl an hybriden Journalen zeigt deutlich, dass es den Verlagen ein großes Anliegen ist, in der Debatte um Open Access eine nicht nur verweigernde Haltung einzunehmen. Vor allem die Hybridlösung ist für Verlage in dieser Hinsicht attraktiv. Dabei ist allerdings ein als Double Dipping bezeichneter Effekt zu bedenken, der vor allem von Bibliotheken und Forschungsförderern als Einwand gegen dieses Modell gebraucht wird. Unter Double Dipping wird die Tatsache verstanden, dass die Verlage sowohl durch Subskriptionsgebühren als auch durch den Freikaufvon Artikeln Einkünfte generieren. Mittlerweile gibt es Initiativen, die diesem Problem entgegenwirken sollen, wie etwa die Preisanpassung der Subskriptionsjournale auf Basis der veröffentlichten hybriden Artikel. Die intendierten Maßnahmen zur Preisanpassung liegen im besonderen Interesse dieses Beitrages. Viele „Big Player“ des Verlagswesens (Elsevier, Wiley, Taylor \& Francis, SAGE, Cambridge University Press, Oxford University Press, American Chemical Society, Nature Publishing Group, Royal 
Society u.a.) bieten die Hybridoption für ihrer Zeitschriften an, die auch genutzt wird ${ }^{1}$. Somit fordern Bibliotheken und Forschungsförderungsorganisationen als eine mögliche Lösung des Problems eine Anpassung der Listenpreise beim hybriden Modell ${ }^{2}$ und mehr Transparenz bei der Durchführung.

Wie Verlage zu diesen Forderungen stehen, inwieweit sie bereit sind, darauf einzugehen und wie sie die Möglichkeiten einer Anpassung sehen, soll nachfolgend dargestellt werden. Die zu Grunde liegende Frage ist, inwieweit es möglich ist, Transparenz in Bezug auf das hybride Zeitschriftenmodell für Bibliotheken, Forschungsförderer, Autorlnnen und Institutionen gleichermaßen herzustellen?

Um dieser Frage nachzugehen, wurden die einzelnen Websites von sieben ausgewählten Verlagen (Springer, Wiley, Taylor \& Francis, Oxford University Press, Elsevier, SAGE, Cambridge University Press) untersucht. Dabei wurden die allgemeinen Informationen zu hybriden Journalen begutachtet. Zusätzlich wurde erhoben, welche Informationen neben Preis und Kennzeichnung von hybriden Journalen auf der Website ansonsten verfügbar sind, insbesondere die Berechnungsgrundlage für die Anpassung der Subskriptionspreise bzw. die im Fall vorhandenen Listen mit Journalen, deren Preise auf Basis der vorhandenen hybriden Artikel gesenkt wurden.

\section{Ergebnisse der webseitenbasierten Analyse zur Preisanpassung bei hybriden Journalen}

Einen prinzipiellen Vergleich zwischen den Verlagsinformationen zu Preisnachlässen auf den jeweiligen Websites zu erarbeiten, gestaltete sich schwierig, da die veröffentlichten Daten nur unzureichend vergleichbar sind. Wenn in der Aufstellung von Springer die Listenpreise und deren Verlauf über die Jahre angegeben werden, liegen zwar wichtige Daten vor, allerdings sind keine weiteren Kennzahlen (wie z.B. die Anzahl der im jeweiligen Journal veröffentlichten hybriden Open Access-Artikel) genannt (vgl. Spinka, 2013). Dementsprechend ist beispielsweise der Preisanstieg um 8\% von EUR 3.559 im Jahr 2011 auf EUR 3.845 im Jahr 2012 bei fast gleich bleibender Anzahl an freigekauften Artikeln im Journal "Cellular and Molecular Life Sciences“ (2012 wurde ein Artikel weniger hybrid veröffentlicht) (vgl. Spinka, 2013) für Außenstehende nicht nachzuvollziehen.

Ähnlich schwer nachvollziehbare Preisentwicklungen gibt es mit Ausnahme von Wiley Blackwell bei allen untersuchten Verlagen. 
Taylor \& Francis gibt zwar die Anzahl der hybriden Artikel sowie den Prozentsatz an, um den der Preis des jeweiligen Journals gesenkt wurde bzw. wird, um der Transparenz allerdings vollständig Genüge zu tun, wäre ebenso eine Bekanntgabe der jeweiligen Journallistenpreise notwendig. Denn wenn acht hybride Artikel im „Journal of Broadcasting \& Electronic Media" eine Reduktion von 6,9\% des Preises bewirken, auf der anderen Seite aber sieben hybride Artikel im Journal „Global Public Health“ zu einer Preissteigerung von 0,6\% führen, dann wäre hier eine Erklärung notwendig (vgl. Taylor \& Francis, 2015). Eine Preissteigerung kann nur nachvollzogen werden, wenn zusätzlich wichtige Referenzzahlen, wie etwa die Gesamtzahl an veröffentlichten Artikeln, genannt werden. Ansonsten kann das Ziel, das mit der Veröffentlichung dieser Zahlen angestrebt wird - die Herstellung von Transparenz - nicht erreicht werden. Vielmehr stellt sich ein Verlust an Glaubwürdigkeit ein. Die Angabe von Referenzen wäre zudem nicht sehr aufwändig, denn die Anzahl der preisangepassten Journale ist mit vier Stück äußerst überschaubar.

Oxford University Press gibt überhaupt keine Zahlen zur Preisanpassung bekannt. Wobei Rhodri Jackson, Verantwortlicher für Open Access bei Oxford University Press, im Interview angab, dass die Preise von Subskriptionszeitschriften auf Basis der freigekauften Artikel gesenkt werden. Gerade beim Journal „Bioinformatics“, das mit über $30 \%$ hybrider Artikel einen sehr hohen Anteil aufweist (vgl. OUP Case Studies, 2015) wären Zahlen zu den erschienenen und freigekauften Artikeln bzw. Preisen aus den Vorjahren und eine Angabe der Preisreduktion in Prozent besonders wichtig.

Wiley Blackwell stellt seine Berechnungsgrundlagen am transparentesten zur Verfügung. Neben den subskribierten Artikeln der letzten beiden Jahre wird auch die Anzahl der freigekauften Artikel der letzten beiden Jahre veröffentlicht (vgl. Wiley, 2015). Zusätzlich wird die tatsächliche Preissenkung bzw. -steigerung pro Journal angegeben. Aus Vollständigkeitsgründen könnten hier noch Angaben zu den Preisen der Jahre 2011 und 2012 bereitgestellt werden. Darüber hinaus wären auch noch die Preise ohne hybride Preissenkung interessant.

Elsevier gibt als einziger Verlag Auskunft über die Gesamtanzahl an freigekauften Artikeln für das Jahr 2013. Insgesamt stehen in jenem Jahr rund 2.000 gesponserte Artikel 330.000 publizierten Artikeln gegenüber (Elsevier, 2015). Außerdem informiert der Verlag noch darüber, in welchem Journal wie viele Artikel freigekauft wurden. Weiterführende Informationen werden aber auf der Website nicht zur Verfügung gestellt. Dem Transparenzanspruch kann dies nicht genügen. Vor allem fehlen Zahlen zu den Preisen von Journalen bzw. deren Preisentwicklung. Würden diese 
bekannt gegeben, wäre die Möglichkeit eines Vergleiches (Preisentwicklung mit hybriden Artikeln) gegeben.

SAGE beschäftigt sich laut Website derzeit mit der Etablierung einer No Double Dipping-Policy. Dies ist auch notwendig, da der Verlag neben Cambridge University Press zu den letzten großen Anbietern gehört, die keinerlei Preisanpassungen vornehmen. SAGE macht keine Angaben dazu, wie diese gehandhabt werden soll (vgl. SAGE, 2015).

Das Gleiche gilt für Cambridge University Press. Laut Verlag wird derzeit aktiv die Entwicklung der Akzeptanz von freigekauften Artikeln beobachtet - mit dem Ziel, eine Methode zur Unterbindung von Double Dipping zu entwickeln (vgl. Cambridge University, 2015).

\section{Empfehlungen zu mehr Transparenz}

Die Masterthesis, die diesem Aufsatz zu Grunde liegt, wurde weitestgehend von der Frage geleitet, inwieweit es möglich ist, Transparenz für das hybride Zeitschriftenmodell bzw. für die damit in Verbindung stehende Preisanpassung zu erreichen. Die Verlagswebsites sind zwar für alle Interessierten gut zugänglich, allerdings finden sich auf jeder der sieben untersuchten unterschiedliche Informationen.

Zur Methode, auf deren Basis der Preis angepasst wird (Anzahl der freigekauften Artikel im Vorjahr bzw. in den Vorjahren), geben bis auf zwei Verlage (SAGE und Cambridge University Press) alle untersuchten Verlage Auskunft. Die Methode ist insofern zulässig, als dass Informationen über die Anzahl der freigekauften Artikel erst am Ende des darauffolgenden Jahres oder zwei Jahre danach (wenn die Möglichkeit des rückwirkenden Freikaufs bedacht wird) ersichtlich sind. Prinzipiell trägt es zur Transparenz bei, wenn eine Liste jener Journale veröffentlicht wird, die einer Neuberechnung unterliegen. Nicht alle untersuchten Verlage tun dies, allerdings würden solche Listen den bestmöglichen Überblick bieten und die Preiskalkulation nachvollziehbar machen - und sind somit unabdingbar. Was diese Listen beinhalten sollten, um für Institutionen, Bibliotheken und Forschungsförderer den größten Nutzen zu bereiten, wird nachfolgend ausgeführt.

Die Berechnungsmethode ist von allen Verlagen klar skizziert. Bei genauer Betrachtung wird allerdings ersichtlich, dass zwar die Herangehensweisen transparent sind, die Berechnungsgrundlagen allerdings nicht.

- Unabdingbar für Transparenz und Überprüfbarkeit ist die Veröffentlichung bzw. übersichtliche Darstellung aller in hybriden Journalen veröffentlichten 
Artikel. Dies kann zurzeit in den meisten Fällen nur mit großem Aufwand nachvollzogen werden.

- Dringend notwendig wäre eine Darstellung der Anzahl der freigekauften Artikel in hybriden Journalen, idealerweise gleich auch im Verhältnis zur Gesamtzahl der im Journal insgesamt veröffentlichten Artikel. Dies kann zwar ebenfalls manuell durch Zählung erfolgen, ist aber in der Praxis aufgrund des hohen Aufwandes nicht durchführbar. Nur mit dem Wissen über die tatsächliche Anzahl an publizierten, nicht freigekauften und freigekauften Artikeln kann eine Preisanpassung nachvollzogen werden. Dies würde auch einen guten Überblick über die Akzeptanz der hybriden Option geben und eventuell die Frage beantworten, ob eine weitere Investition in dieses Modell (z.B. vonseiten der Forschungsförderer bzw. der Autorlnnen) sinnvoll ist. Zudem ist die Anzahl an freigekauften Artikeln insofern interessant, als dass damit ein Indikator für die Akzeptanz der hybriden Option in den einzelnen Fachgebieten ermittelt werden könnte.

- Als weitere wichtige Komponente zur Transparenz dient der Überblick über den Preisverlauf eines Journals. Nur wenn die Preise der Vorjahre bekannt sind, ist ein Vergleich überhaupt möglich. In diesem Zusammenhang ist die oben angeführte Zahl der Gesamtartikel in einem Journal nochmals zu erwähnen. Durch Anführung dieser und deren Entwicklung in den Jahren davor kann eine mögliche unterdurchschnittliche Preissteigerung oder Senkung schnell festgestellt werden. Sollte ein Journal mehr Artikel veröffentlichen, ist klar, dass der Arbeitsaufwand und folglich der Preis des Journals steigt. Hier wäre vor allem aber auch eine klare Trennung einzelner Preisfaktoren vonnöten; es sollte nachvollziehbar sein, welcher Faktor ein Journal billiger bzw. teurer macht; z.B.: 1) Ein Journal wird aufgrund von Freikäufen um x Euro billiger und 2) ein Journal wird aufgrund der gestiegenen Zahl an veröffentlichten Artikeln um y Euro teurer. Im Moment ist diese Übersichtlichkeit nicht gegeben. Dies führt zwangsläufig zu Annahmen und Vermutungen vonseiten der Bibliotheken, Autorlnnen und Forschungsförderer, die allerdings keine klaren Aussagen zulassen.

- Hilfreich wäre auch die explizite Angabe der prozentuellen Preisänderung eines Journals. Neben der offensichtlichen Informationsfunktion dient diese Angabe auch als Referenzzahl und Richtwert. Dadurch ist erkennbar, wie viel der einzelne Artikel im Journal wirtschaftlich wert ist. 
- Darüber hinaus wäre in weiterer Folge interessant zu erfahren, bei welchem Wert die Schwelle Anzahl/Prozentsatz an Artikeln, die freigekauft werden müssen, damit ein Journal günstiger wird liegt, falls nicht schon ein einzelner hybrider Artikel zu einer Preissenkung des Journals führt. Taylor \& Francis gibt beispielsweise darüber Auskunft, dass ab einer Anzahl von fünf freigekauften Artikeln das gesamte Journal günstiger wird, wenn diese fünf Artikel mehr als $5 \%$ des gesamten Inhaltes ausmachen. Die anderen Verlage bieten darüber keine Information. Vielfach wird der Anschein erweckt, dass bereits ein freigekaufter Artikel zu einer Vergünstigung führt. Aus den im nachfolgenden Kapitel vorgestellten Befragungen geht allerdings hervor, dass dem nicht so ist. Ein Verlag gab beispielsweise an, dass der Schwellenwert in seinen Journalen bei $8 \%$ des Inhalts liegt. Hervorzuheben ist hierbei, dass der Verlag dies nicht auf seiner Website publiziert.

- Als Vergleichswert wäre außerdem auch der Preis interessant, der für ein Journal zu bezahlen wäre, wenn keine Artikel freigekauft werden.

\section{Befragung der Verlage und Ergebnisse}

Im Zeitraum Oktober bis Dezember 2013 wurde eine qualitative Umfrage mit Hilfe des Websurvey-Tools "Google Drive“ bei den sieben Verlagen (Springer, Taylor \& Francis, Oxford University Press, Wiley Blackwell, Elsevier, SAGE, Cambridge University Press) durchgeführt, um den Standpunkt sowie die Haltung von diesen Verlagen zum Thema hybride Journale festhalten zu können. Die Kriterien zur Auswahl der Verlage waren:

- Verlage unterschiedlicher Größe

- Gutes Renommee in der Verlagsbranche

- Vorhandenes Angebot von hybriden Journalen

- Nutzung der Option Hybrid vonseiten der AutorInnen

- Informationsangebot zum Thema auf der Website

- Vorhandensein von Kontaktpersonen für hybride Journale bzw. Open Access

Von den sieben befragten Verlagen haben vier geantwortet (Rücklaufquote $57 \%$ ). Trotz mehrmaliger Einladung zur Teilnahme an der Befragung beantworteten drei Verlage den Fragebogen nicht. Im Hinblick auf die Ehrlichkeit und Reichhaltigkeit der Antworten wurde den Verlagen versichert, dass die Antworten anonymisiert wiedergegeben werden. 
Der Fragebogen war in sechs Themenblöcke gegliedert:

1. Nutzung und Wahrnehmung sowie Gebrauch von hybriden Journalen

2. Sichtbarkeit von Open Access auf der Website

3. Haltung zu Double Dipping sowie Möglichkeit der Transparenz

4. Anpassung der Subskriptionspreise und ihre möglichen Varianten

5. Zukunft von Open Access - weitere Entwicklungen

6. Verwaltung von APCs

Der Online-Fragebogen umfasste 17 Fragen, mit denen ermittelt werden sollte:

- warum hybride Journale angeboten werden;

- wie der jeweilige Verlag die Möglichkeiten für dieses Geschäftsmodell in Bezug auf Wachstum, Verbreitung und Akzeptanz bei den Autorlnnen einschätzt;

- wie hoch die Nutzung der Option Hybrid ist;

- wer für die Artikelgebühren aufkommt;

- wie die Position zum Thema Double Dipping ist;

- ob vonseiten des Verlags Initiativen zur Verhinderung von Double Dipping ergriffen werden;

- ob es eine Richtlinie zur besseren Transparenz zum Thema Double Dipping gibt;

- wie der präferierte Weg der Kostenanpassung ist;

- warum im Falle einer Reduktion der Subskriptionspreise dies nicht stärker publik gemacht wird;

- ob angedacht ist, die Anzahl und Titel von hybriden Artikeln auf der Website zu publizieren;

- in welchem Ausmaß die Wandlung von hybriden Journalen zu reinen Open Access Journalen geplant ist;

- wie viele freigekaufte Artikel benötigt werden, um den Threshold zu erreichen, ab dem eine Rückvergütung möglich ist;

- ob überlegt würde, aus einem Journal ein Open Access-Journal zu machen, wenn in diesem mehr als $51 \%$ des Inhaltes freigekauft würden;

- ob das hybride Modell ein wichtiges Element in der weiteren Strategie des Verlages darstellt;

- ob das Management der Article Processing Charges ausgelagert würde;

- ob für einen solchen Service bezahlt werden würde; und wer in dieser Hinsicht die präferierten PartnerInnen wären. 


\subsection{Nutzung und Wahrnehmung sowie Gebrauch von hybriden Journalen}

Alle Verlage antworteten hier, dass sie sich auf Druck ihrer Autorlnnen dazu entschlossen hätten, die hybride Variante anzubieten. Dies zeigt deutlich, dass Verlage ihre wirtschaftliche Vorgehensweise nicht alleine bestimmen können, sondern sehr wohl ihren Autorlnnen verpflichtet sind ${ }^{3}$. Somit kommt den Autorlnnen von wissenschaftlicher Literatur eine Schlüsselposition zu, die es in Hinblick auf Open Access zu nutzen gilt. Bezüglich der Frage nach Umsatzwachstum, Verbreitung und Akzeptanz antworteten die Verlage unterschiedlich: während einer die unterschiedliche Unterstützung durch Forschungsförderer in den einzelnen Fachbereichen hervorhob, verwies ein anderer darauf, dass das hybride Modell ein maßgeblicher Teil des Businessmodels seines Verlages sei. Ein dritter antwortete, dass die weitere Entwicklung von Open Access stark von den Mandaten der Forschungsförderungsinstitutionen beeinflusst werde. Somit zeigte sich in den Antworten der Verlage, dass die Verlage durch das vorgegebene Ziel - freier Zugang zu wissenschaftlichen Ergebnissen - von gesetzlicher bzw. Forschungsfördererseite gezwungen sind, alternative Optionen zu bieten, um ihre geschäftlichen Zielvorgaben zu erreichen. Björk und Solomon schreiben in ihrer Studie „Developing an Effective Market for Open Access Article Processing Charges", dass sich Verlage außerdem nicht mehr an den von Springer vorgegebenen USD 3.000 pro Artikel orientieren, sondern die APCs für die hybride Option reduzieren, auch um die Nutzung dieser Option zu erhöhen. Alle Verlage gaben bei der Umfrage an, dass die Nutzung bei 0-10\% liege.

\subsection{Sichtbarkeit von Open Access auf der Website}

Auf die Frage, warum die Anpassung der Subskriptionspreise nicht stärker publiziert wird, verwiesen alle Verlage auf Ihre Websites. Für die Webseitenbesucherlnnen ist es relativ schwierig, die von den Verlagen zur Verfügung gestellten Listen zu finden. Darüber hinaus ist es überraschend, dass für die Reduktion der Subskriptionspreise nicht mehr Werbung gemacht wird. Denn das würde den Autorlnnen eine Möglichkeit bieten, zu erfahren, wie verantwortungsbewusst mit ihren geleisteten Mitteln umgegangen wird. Sie sind in diesem Fall die EntscheidungsträgerInnen.

Zwei Verlage verwiesen bei der Beantwortung der Frage auf die Kennzeichnung von hybriden Artikeln auf ihrer Website. Dies ist überraschend, da sich für die Websitebesucherlnnen das Auffinden der Open AccessArtikel nicht einfach gestaltet. Allerdings könnte durch einheitliche oder individuelle Open Access-Icons über eine Verlagsplattform zielgerichtet 
beim richtigen Publikum Sensibilität für die Thematik geschaffen werden. Würden die Open Access-Icons auf breiter Basis verwendet werden, wäre vielen Autorlnnen bewusst, dass Open Access, egal in welcher Form (Hybrid oder Gold), unter anderem die Reichweite eines Artikels erhöht. Dass, wie von einem Verlag im Interviewteil bezweifelt wird, von Autorlnnen nicht nach Open Access-Inhalten gesucht wird, widerlegen die Suchoptionen bei Elsevier, der Royal Society of Chemistry und bei Cambridge University Press, die die NutzerInnenbedürfnisse erkannt und auf diese reagiert haben.

\subsection{Haltung zu Double Dipping sowie die Möglichkeit der Transparenz}

In diesem Themenkomplex verwiesen die Verlage auf die vorhandenen Preisnachlasslisten sowie auf die Double Dipping Policies auf den jeweiligen Websites. Ein Verlag verwies explizit darauf, dass seit 2011 Mechanismen implementiert worden seien, die Double Dipping verhindern würden und deren Resultat die Reduktion der Preise von Subskriptionsjournalen sei.

Überraschend war die Antwort von einem Verlag, der bekanntgab, dass seine Preisanpassungsliste stark kritisiert wurde. Hier wäre interessant gewesen, zu erfahren, von wem diese Kritik kam und welche speziellen Kritikpunkte es gab.

\subsection{Anpassung der Subskriptionspreise und ihre möglichen Varianten}

Alle Verlage wählten hierbei die Antwort „Auf Basis der Subskriptionen (Reduktion der Subskriptionspreise)“. Nie genannt wurden „Auf nationaler Basis“, „Institutionelle Rückvergütung“, „Tokens“ oder „Sonstiges“. Dass die Verlage durchgehend die Antwortmöglichkeit „auf Basis der Subskriptionen (Reduktion der Subskriptionspreise)" wählten, war nicht überraschend. Diese Option ist bei derzeitiger Handhabung (in Form von Preislisten) allerdings nach wie vor sehr intransparent. Hier fehlen Regeln und Vorgaben, nach denen die Listenveröffentlichung handzuhaben wäre.

\subsection{Zukunft von Open Access - weitere Entwicklungen}

Open Access ist eine Strategie, die laut der Antworten der Verlage in Zukunft weiterverfolgt wird ${ }^{4}$. Darüber hinaus sind auch die hybriden Journale fest im Repertoire der Verlage verankert. Erstaunlich ist, dass die Frage nach der Schwelle der benötigten Artikel von nur einem einzigen Verlag explizit mit einer Zahl beantwortet wurde. So gab dieser Verlag an, dass 
ab einem Treshhold von $8 \%$ bezahlter Artikel und darüber hinaus der Preis angepasst würde. Dies und die anderen Antworten legen nahe, dass es selbst innerhalb eines Verlages keine einheitliche Strategie bezüglich freigekaufter Artikel gibt, bzw. dass dies tatsächlich auf Journalebene entschieden wird. Aus Transparenzgründen wäre es notwendig, zu erfahren, welche Referenzen entscheidend sind und welche Methode je Journal angewendet wird.

\subsection{Verwaltung von APCs}

Zwei der befragten Verlage entwickeln derzeit eigene Verwaltungsmodelle für APCs. Einer hat dies schon getan. Ein einziger Verlag könnte sich das Outsourcen von APCs vorstellen. Die Verwaltung von APCs ist allerdings eine Schlüsselstelle. Wenn ein Dritter, beispielsweise eine Konsortialstelle oder eine Agentur, diese betreut bzw. die Abwicklung der Verrechnung übernimmt, kann unter diesen Umständen mehr Transparenz geschaffen werden. So eine übergeordnete Instanz könnte objektive Informationen bereitstellen, während Bibliotheken und Forschungsförderer bei der Abwicklung der APCs über die Verlage von deren Bereitschaft zur Freigabe der Informationen abhängig sind.

\section{Zusammenfassung}

Der Übergang zu Open Access ist ein andauernder Prozess. Zukünftig wird es wichtig sein, zu eruieren, inwieweit neben Verlagen auch andere Akteurlnnen die Entwicklung beeinflussen können. Meiner Ansicht nach sind hier vor allem die Forschungsförderer sowie staatliche Einrichtungen aufgefordert, Richtlinien zu entwickeln, die die Transparenz der verwendeten Steuermittel gewährleisten. Die Untersuchung und Befragung der Verlage hat gezeigt, dass es derzeit keine echte Kostentransparenz gibt. In diesem Zusammenhang stellt sich natürlich die Frage, inwieweit die hybride Variante (die letztendlich zu mehr Open Access führt) finanziell von Forschungsförderern unterstützt wird, wenn es zu keinem transparenten Kostenausgleich kommt.

Mag. a Sabine Stigler, Bakk. MSc EBSCO Information Services GmbH

Ignaz-Köck-Str. 9, A-1210 Wien

E-Mail: sstigler@ebsco.com 


\section{Literatur}

Björk, B.-C. (2012). The Hybrid Model for Open Access Publication of Scholarly Articles: A Failed Experiment? Journal of the American Society for Information Science and Technology Vol. 63, Iss. 8, p. 1496-1504. doi:10.1002/asi.22709

Björk, B.-C. \& Solomon, D. (2014). Developing an Effective Market for Open Access Article Processing Charges. Abgerufen am 22. 2. 2015 von Wellcome Trust: http://www.wellcome.ac.uk/stellent/groups/ corporatesite/@policy communications/documents/web document/ wtp055910.pdf

Cambridge Journals Online. (2014b). Abgerufen am 22. 2. 2015 von Open Access publishing at Cambridge: http://journals.cambridge.org/ action/displaySpecialPage?pageld $=4604 \#$ Readers

Elsevier. (2015). Abgerufen am 22. 2. 2015 von No Double Dipping Policy Elsevier: http://www.elsevier.com/about/open-access/open-accesspolicies/no-double-dipping-policy

FWF. (2014a). Abgerufen am 22. 2. 2015 von Jahresbericht 2013: http:// www.fwf.ac.at/de/public relations/publikationen/jahresberichte/fwfjahresbericht-2013.pdf

FWF. (2014b). Abgerufen am 22. 2. 2015 von FWF- DerWissenschaftsfonds Aktuelles: http://www.fwf.ac.at/de/aktuelles detail.asp?N ID =587

Oxford University Press. (2015). Abgerufen am 22. 2. 2015 von Oxford Journals Oxford

Open Case Studies:http://www.oxfordjournals.org/en/oxford-open/casestudies.html

SAGE. (2015). Abgerufen am 22. 2. 2015 von SAGE Choice: http://www. sagepub.com/sagechoice.sp

Spinka, V. (2013). Springer Open Choice Price Adjustment 2014. Abgerufen am 22. 2. 2015 von Springer: http://static.springer.com/ sgw/documents/1418628/application/pdf/Springer+Open+Choice Journal+Price+Adjustments+2014.pdf

Taylor \& Francis online. (2014). Abgerufen am 22. 2. 2015 von Taylor \& Francis online - Open Access - Funders \& Institutions: http://www. tandfonline.com/page/openaccess/funders

Wiley Online Library. (2013). Abgerufen am 22. 2. 2015 von Wiley Online Library - Subscription prices for hybrid journals: http://olabout.wiley. com/WileyCDA/Section/id-816521.html 
1 So hat der der Fonds zur wissenschaftlichen Forschung (FWF) im Jahr 2013 um EUR 2,1 Millionen Artikel hybrider Zeitschriften freigekauft (vgl. FWF 2014a, S. 72).

2 Dies ist nur eine Möglichkeit, wie auf das Problem Double Dipping lösend eingegangen werden kann. Darüber hinaus gab es im Jahr 2014 eine Einigung (Nationale Rückvergütung) zwischen dem Wissenschaftsverlag Institute of Physics, der Zentralbibliothek für Physik, der Kooperation E-Medien und dem FWF: dieses Abkommen beinhaltet, dass der FWF Kosten für die Open Access-Freischaltung übernimmt, diese werden wiederum den österreichischen Forschungsstätten von ihren Subskriptionspreisen abgezogen (vgl. FWF, 2014b). Ein weiteres Modell stellt das „Gold for Gold“-Programm der Royal Society of Chemistry dar. Dies sieht die Ausschüttung von Gutscheinen zur Publikation von Open Access-Artikeln beim Kauf des RSC Gold-Pakets vor.

3 Auch Björk und Solomon gehen davon aus, dass bei der Entscheidung, wo publiziert werden soll, nicht nur der Preis der APCs entscheidend ist, sondern auch die Qualität der Betreuung (Björk \& Solomon, 2014).

4 Dies zeigt sich auch dadurch, dass immer mehr Verlage, die auf Subskriptionsjournale fokussiert waren, nun ihre eigenen OA Journale auf den Weg bringen. Außerdem werden Subskriptionsjournale auf OA Journale geändert, so zum Beispiel im Fall von Elseviers „Stem Cell Research" (vgl. Björk \& Solomon, 2014).

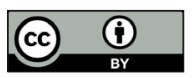

Dieses Werk ist lizenziert unter einer Creative-Commons-Lizenz Namensnennung 3.0 Österreich. 